\title{
Artistically Conveying Peripheral Information with the InfoCanvas
}

\author{
Todd Miller \\ College of Computing/GVU Center \\ Georgia Institute of Technology \\ Atlanta, GA 30332-0280 USA \\ $+14043852447$ \\ tomiller@cc.gatech.edu
}

\author{
John Stasko \\ College of Computing/GVU Center \\ Georgia Institute of Technology \\ Atlanta, GA 30332-0280 USA \\ +14043852447 \\ stasko@cc.gatech.edu
}

\begin{abstract}
The Internet and World Wide Web have made a tremendous amount of information available to people today. Taking advantage of and managing this information, however, is becoming increasingly challenging due to its volume and the variety of sources available. We attempt to reduce this overload with the InfoCanvas, an ambient display of a personalized, information-driven, visual collage. Through a web-based interface, people identify information of interest, associate a pictorial representation with it, and place the representation on a virtual canvas. The end result is an information collage, displayed on a secondary monitor or net appliance, that allows people to keep tabs on information in a calm, unobtrusive manner. This paper presents details on how a person can create and manage information with the InfoCanvas, and how we provide such capabilities.
\end{abstract}

\section{Categories and Subject Descriptors}

H.5.2 [Information Interfaces and Presentation]: User Interfaces - Graphical User Interfaces, interaction styles.

\section{General Terms}

Design, Human Factors

\section{Keywords}

Information awareness, ambient display, peripheral information display, visualization

\section{INTRODUCTION}

The Information Age, largely facilitated by the Internet, has brought with it an overwhelming amount of data. ${ }^{1}$ Today, it is possible to quickly access information on a tremendous variety of topics. One particular class of information includes data that is not absolutely essential to people in their work or tasks, but that is often very helpful and useful. People often monitor this type of information intermittently throughout a day. Examples of this include weather forecasts, traffic conditions, financial statements, auction prices, news stories, current airfares, sports scores, and email. We term this data "peripheral awareness information" and it is the focus of our work.

Monitoring peripheral awareness information has arguably become a part of daily life for most people working with information technology. Some people will access this type of information at the beginning or end of a workday, or even during breaks. Others intermittently examine information throughout the day. Unfortunately, this activity can be distracting and disruptive to other important tasks. As the amount of information available to us continues to increase, tools that allow us to easily monitor and filter it will become increasingly important. However, the current means for monitoring this data has been fairly limited and/or time consuming. The common approach is to realize the desire to see the information, start a web browser, access the page with the desired information, mentally process it, and then resume a previous task. This process is heavyweight in terms of cognitive workload. A person can easily forget to check information since it requires their explicit attention. In addition, some people might choose not to check the data because the time required reduces the overall benefit.

\subsection{Desktop Overload}

If someone were to try to monitor a great deal of peripheral awareness information, their desktop would quickly become cluttered and might resemble something similar to that illustrated in Figure 1. The person's display is littered with stock quotes, traffic reports, to-do lists, unread emails, news headlines, weather forecasts, and instant messengers. All of this information leaves virtually no room for actual work to be performed.

In an attempt to find easier ways to manage all of this information, people have developed a variety of techniques and tools. One simple approach is to use the Windows Active Desktop to display a collage of web pages, allowing the user to keep a variety of information displayed in the background of their workspace. However this requires users to minimize all their windows in order to see the data on the desktop. Personalized portal pages, like that of MyYahoo! [8], allow people to consolidate some of the information into one web page making it easier to manage. However, the user is limited in the data that can be monitored and the method in which it is displayed. Another solution is to use a second monitor for displaying the peripheral information, an increasingly common approach used by those with two display devices [2]. Some attempts have also been made to move the data off of the computing desktop to the physical environment through the use of visual and aural effects, thus allowing users to save their virtual workspace for their primary tasks [11]. We believe that a combination of the best aspects of each of these approaches creates an effective tool for monitoring

\footnotetext{
${ }^{1}$ Lyman and Varian estimate that over 1.5 billion gigabytes of data is produced each year, with $80 \%$ of that being in electronic form [5].
} 


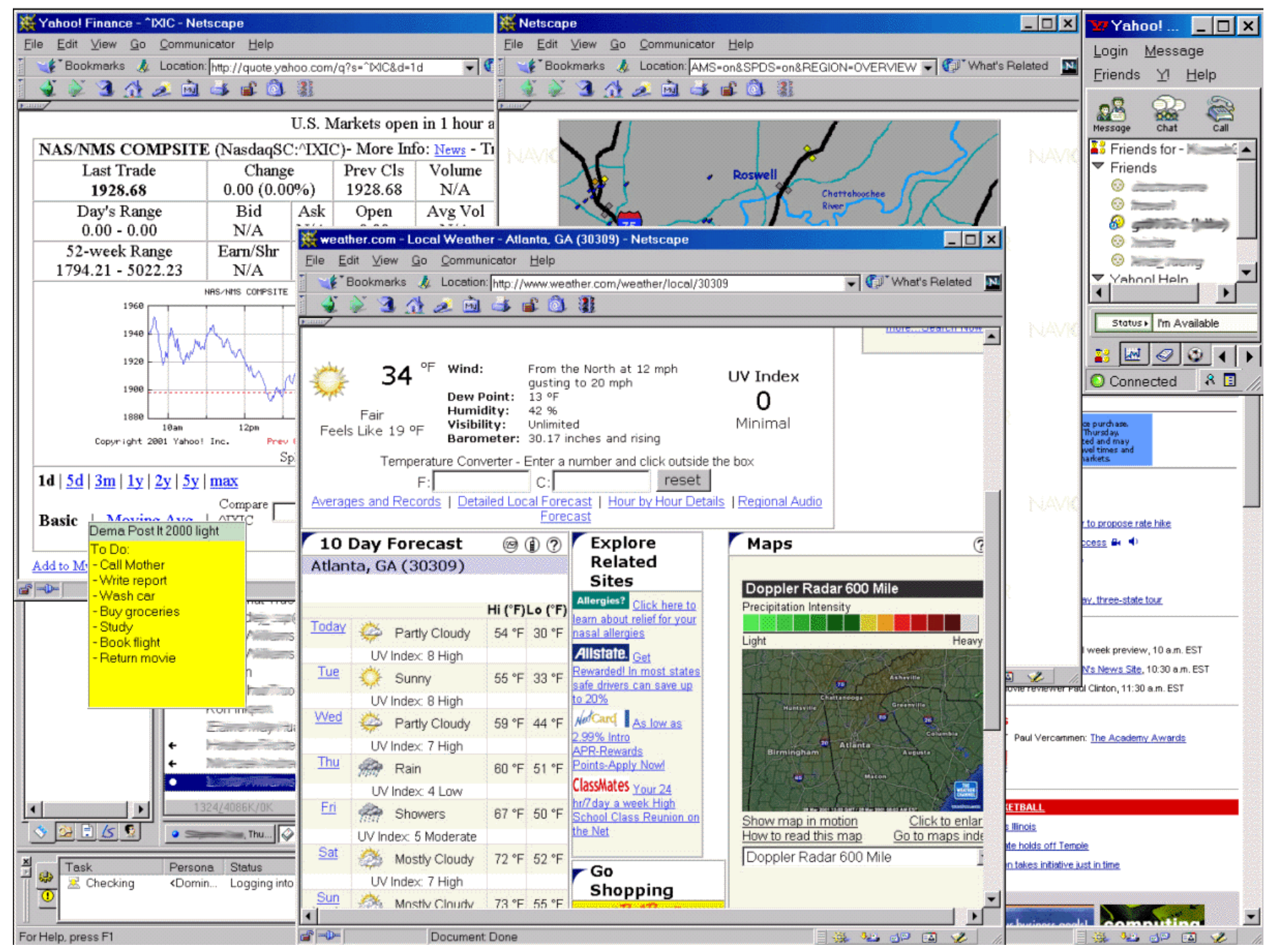

Figure 1. An overloaded desktop

peripheral awareness information. In combining the best elements of the different methods of dealing with information, we propose

1) Available. It should be always available and easily accessible. The overhead of starting a web browser and loading a web page reduces the usefulness of the system because it prevents the user from being able to quickly obtain information without any delay. In addition, the data should always be current within an acceptable time frame for the type of data being monitored - as users will have an expectation that such a display will portray relatively up-to-date information.

2) Consolidated. It should consolidate information from a variety of sources, allowing a variety of data to be conveyed through one display. Providing the user with a complete and integrated view will increase the overall utility of the system.

3) Peripheral. The representation should be calm and peripheral in nature [10]. Peripheral awareness information, by definition, is not information that should demand attention from the user. Therefore, a display should use little or no animation and not draw attention to itself when updating.

4) Personalized. The display itself should be personalized to the user. Allowing the user to monitor virtually any data in the manner of their choosing enables them to create a representation that is more meaningful to them, reducing the time it takes to process the data displayed. Restricting the user to specific types of the following five properties that a tool for monitoring peripheral awareness information should exhibit.

data or a single method of representation reduces the utility of the display and makes it difficult to achieve the property of information consolidation.

5) Secure. Displays should hide sensitive or personal data, guarding the privacy of the user. Some peripheral information is very sensitive as well as very desirable to monitor, such as bank account balances. A display should afford monitoring such information in a manner that is not overly revealing. This is particularly important in displays that are used in public or semipublic settings such as offices, Internet cafes, or lab settings. An overly direct representation of data on the display will reduce the user's willingness to portray all of the information that is important to him or her.

\subsection{The InfoCanvas}

In an effort to create a tool that exhibits all five of these properties, we developed the InfoCanvas. The InfoCanvas is a pictorial representation of a variety of information sources that is designed to be presented on a second display that sits on a desk like a picture frame (see Figure 2) or a painting hung on a wall (see Figure 3). This allows the user to glance at the display and quickly obtain the status of a variety of information. 


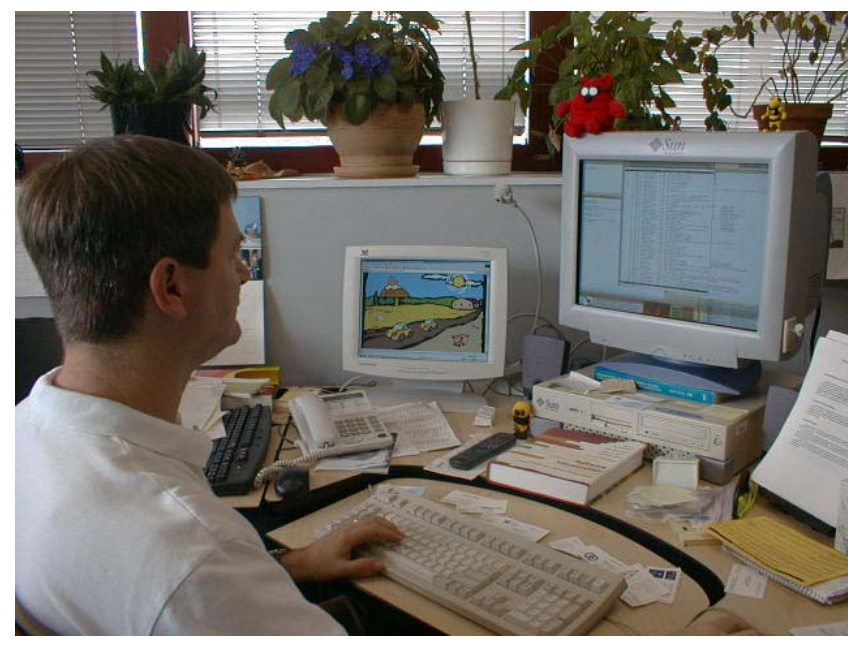

Figure 2. The InfoCanvas as a desk picture frame

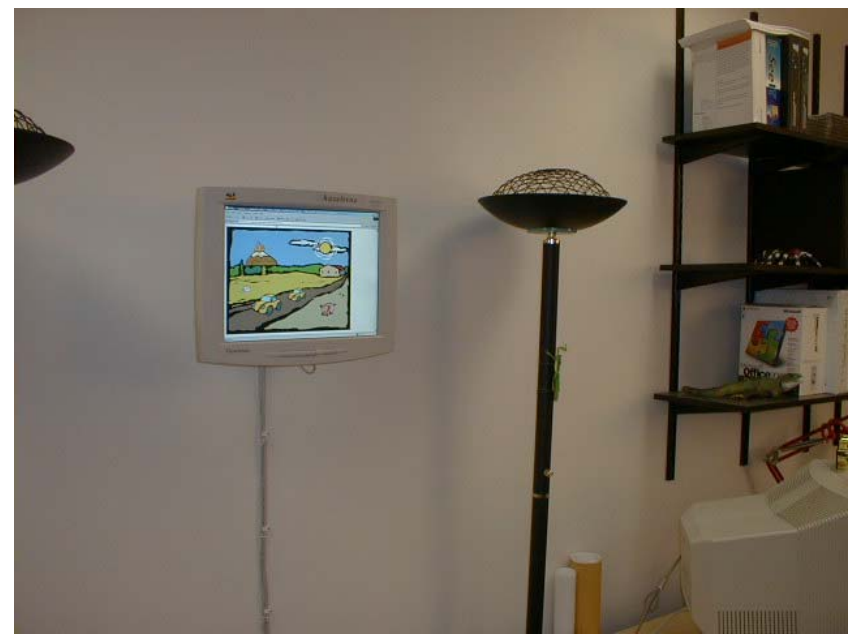

Figure 3. The InfoCanvas as a painting hung on a wall

The InfoCanvas exhibits all five of the proposed properties. It is always available and easily accessed by the moving the display to a secondary device. It is also possible to run it as a desktop application that the user can switch to when they desire the information their canvas represents. The InfoCanvas can monitor information from web pages and emails, creating a collage of data, consolidating the information in one place. The user is given ultimate control in deciding what information is to be monitored and how it is to be represented, allowing them to design highly personalized displays. The abstract nature of the representation allows us to convey the data in a peripheral manner. Subtle changes are made to the picture as the underlying data changes, as opposed to tickering text or cycling through paragraphs. This also lends itself to security, since it eliminates text and numbers that could be overly revealing.

The current implementation of the InfoCanvas is designed to be used as a personal information monitoring tool and is managed through a web-based interface. Since our main goal is to explore the possibilities and problems of such a tool, we have created a simplified initial version, limiting both the information that can be monitored and the ways it can be represented on the canvas. This article reports on this initial implementation. However, a fullfeatured version of the InfoCanvas would allow the user to be as artistic as they desire, letting them "paint" virtually any information they wish onto the canvas. An early glimpse of that vision was presented at CHI 2001 [7].

\section{INFORMAL STUDY}

To gain a better understanding of the type of information people currently monitor and the way that they do it, we conducted informal interviews of twenty-five people. We asked each participant questions about the types of information that they check regularly, when they check it, and what is important to them about it. The subjects covered a wide range of ages, from 23 to 60 , and backgrounds including secretaries, a house wife, college students, computer professionals, marketers, and entrepreneurs.

In general, we found that $80 \%$ of our participants check news and financial web sites on a regular basis; however there was great diversity overall among the responses as to which sites or type of information specifically. Most participants indicated that they would check a set of web pages once a day or so, usually when they first arrived at work or before they left. However, four people responded that they would check on information repeatedly throughout the day, sometimes using it as a break between tasks. Two participants commented that they would occasionally choose not to check on some information, or decide to wait until later and possibly forget about it all together, because it was not convenient to access it at the time they thought about it.

In general, we found that most of the subjects were somewhat habitual in their own browsing of the web. When asked what sites they would visit on a regular basis, all of them were able to quickly list off half a dozen or so. Some tended to focus on news and events, while others used the web for finding bargains on goods and services, while others still were mainly interested in keeping in touch with friends and family. A majority (56\%) of the participants used some sort of "start page", such as MyYahoo!, as their home page. The most cited reason for this was that it is an easily available tool for integrating a variety of personally relevant information.

Overall, this confirmed our belief that this type of information is important and is desirable to people to monitor. We learned that the information people want to monitor is about as varied as the people themselves, and thus a great deal of flexibility is needed in supporting this type of activity. This emphasizes the property of personalization and its importance in designing awareness tools. In order to better assist people with monitoring, a larger study is warranted to further explore the browsing habits to develop typical profiles of web use in relation to peripheral information.

\section{PERIPHERAL INFORMATION}

Based upon the important aspects of the data that people monitor, we realized that there are two fundamental modes of representation: unconditional and conditional. The mode is determined by what aspect of the data is important to the user. The modes of representation affect the interface of InfoCanvas, or any other monitoring tool, in both how the user specifies what is to be watched and how it is to be represented. In this section, we discuss these two modes and their effects on InfoCanvas. 


\subsection{Unconditional}

In some situations, it is desirable to always show the current state of a piece of information. Representations of this type come under the unconditional mode. Examples include depicting weather forecasts and news updates.

Information that is always present can only convey a change in status to the user by changing its pictorial representation. Indicating a change may mean moving it to a different location, changing its size or color, using different icons, or some other transformation. The variety of possibilities creates a need for some form of end-user programming or specification, but allowing users to easily program a representation for their data is difficult.

\subsection{Conditional}

This type of data is only of value when the data itself satisfies an associated condition. For example, a user might want to be notified when they receive an e-mail from a particular person, when a price falls below (or rises above) a particular threshold, or when a web page has been updated.

This information can also be time-sensitive in nature, in which it is only desirable to represent it during certain times of the day, week, or month. For example, the local traffic condition is primarily important when the user is about to drive somewhere, and the score of a baseball game is most valuable while the game is being played and for a day or two afterwards.

The boolean nature of this mode leads to fairly simplistic representations - an item is either present on the display or it is not. The end-user programming problem of an unconditional representation can be avoided, as all the user needs to specify is an icon. However, it introduces the need to allow the user flexibility in specifying how the information is to be harvested and the criteria for it to be shown on the display. This effectively creates another need for end-user programming of the display.

It is also important to note that the mode of representation depends on how the information is to be used. For example, if a person only wants to know when the weather forecast calls for rain, then the mode would be conditional rather than unconditional. Thus, personal preferences and needs will determine how and when data is represented on the display.

\section{CREATING AN INFOCANVAS}

In this section, we describe the process through which a user can create and manage a canvas. The current version utilizes a webbased approach, allowing users to view and control their InfoCanvas from anywhere. Each canvas consists of a background scene, such as a landscape or still-life, with data-driven elements layered on top. Initially, the canvas is completely blank with no data represented. It is left to users to decide what they want to place onto their canvas.

Each element of the scene depicted on the InfoCanvas is driven by data identified by the user. For example, a user could start with a simple landscape scene. They could then associate the daily stock market performance with the weather shown in the landscape, with clear skies meaning good performance, or a storm indicating the markets are down. Local traffic conditions could be mapped, in a more direct representation, to the number of cars on a road. A piggy bank could expand or contract in size as their bank account balance changes. When an e-mail is received from their boss, a volcano in the background could look like it had erupted. Whenever a news headline about their favorite baseball team is found, a baseball could be set to appear over a field. In the end, their canvas might resemble something similar to that of Figure 4. Below, we provide more details about the process of specifying information to monitor and its corresponding representation.

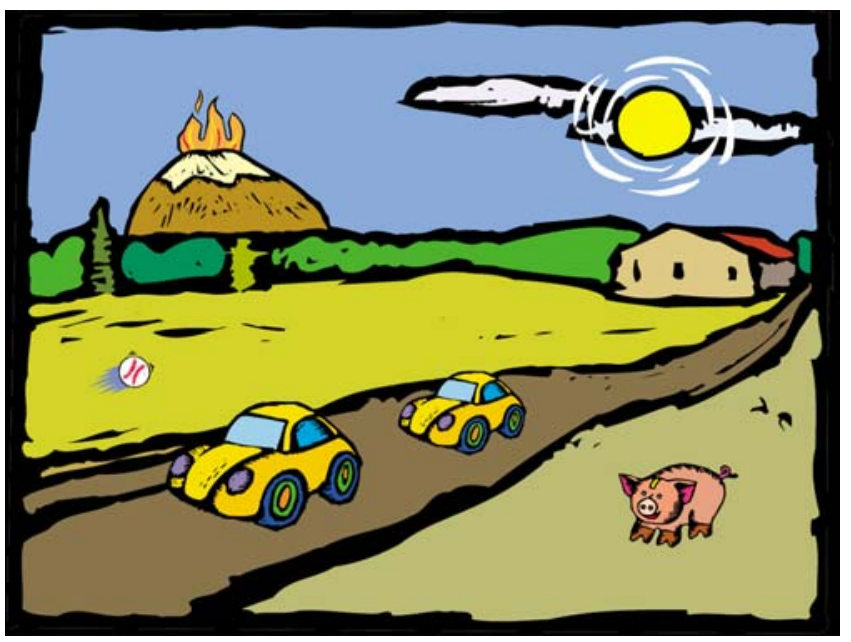

Figure 4. An example InfoCanvas

\subsection{Specifying Information}

The first step in representing information on a canvas is to identify the data to be monitored. Once the user has logged into the InfoCanvas web manager, they will select an "Add Data" operation to specify a data item to be represented on their canvas. This will present the user with a list of the types of information that they can monitor. We initially included the following set of pre-defined information types:

- Weather forecasts

- $\quad$ Stock quotes

- News headline alerts

- Auction price watch

- New search results watch

- $\quad$ Email notifications

- $\quad$ Traffic conditions

- Web page update alerts

- $\quad$ Airfare price watch

- Bank account balance monitor

Once the user selects a type of information of interest, they are prompted for additional data needed for harvesting the actual information, such as a geographical location for the weather or the stock ticker symbol for a stock quote. The user also is asked to give this data a personal label for easier reference, such as "Atlanta weather forecast." The label is used in managing existing data representations so that a user can easily find the data they wish to modify. Also at this point, the user is given the ability to specify times of the day and days of the week for which the information should be shown on the canvas. By default, the 
information will not be restricted by any time constraints. Figure 5 depicts the basic canvas manager web page for a newly created canvas, just after the user has chosen their favorite background image. The user is able to pick information to add from a list of radio buttons.

\begin{tabular}{|l|}
\hline InfoCanvas of Todd Miller \\
Information Manager \\
\hline Canvas Preview \\
\\
Nothing \\
Select information to monitor with your canvas: \\
\\
Airfare watch \\
Auction price watch \\
0 New e-mail notification \\
News headline watch \\
Search results monitor \\
Stock price \\
Today's weather forecast \\
Traffic conditions \\
Web page update alert \\
Add to Canvas \\
\hline
\end{tabular}

Figure 5. The basic InfoCanvas manager screen with a newly created blank canvas shown

\subsection{Associating a Representation}

Once the information has been specified, the next step is to define the pictorial representation of it. For simplicity, each information source comes pre-defined with several generic representations. Each of these generic representations defines a general mapping of data to a visual image. While the mapping is pre-defined, the actual icon(s) used for the image can be chosen by the user. For example, when associating a representation with a weather forecast, the user can choose to show the forecasted condition by icons, the forecasted high temperature by a dynamic thermometerlike icon, or present an icon when only inclement weather is forecast. Once the user has selected one of these three possible representations, they can then choose the icon(s) to go along with the mapping they selected. The icon selection process is shown in Figure 6, which gives the user an overview of the information to be monitored, and allows them to associate images with certain attributes of the data. The user can find or create their own images to use on the canvas and add them to the library through an import feature. This allows the user greater creativity when designing their display than when confining them to a pre-defined representation.

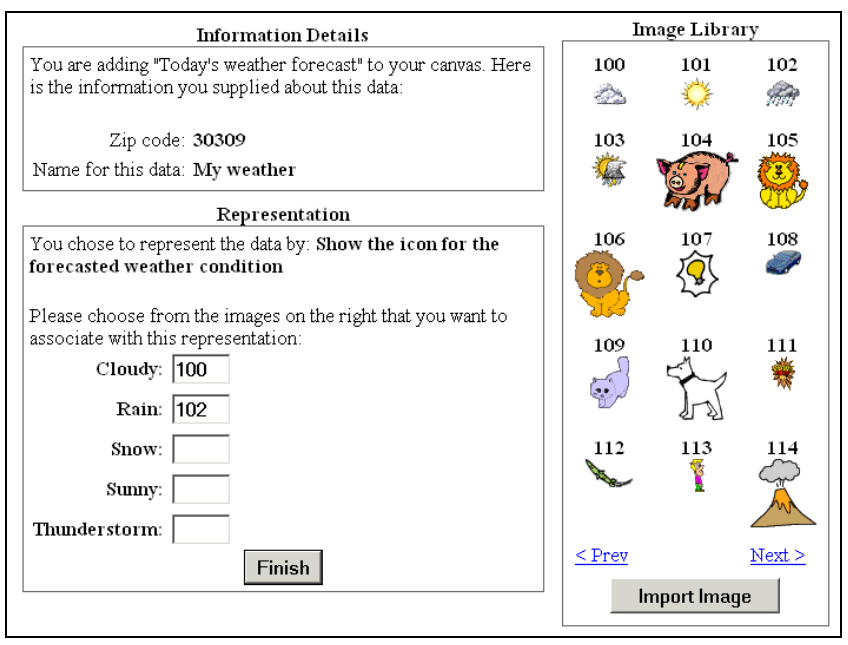

Figure 6. Specifying images to be associated with information being monitored

\subsection{Placement}

The last step in adding new information to the InfoCanvas is placing the chosen representation on the canvas. This is done by previewing a default placement for the representation on the canvas, allowing the user to move it around or change its size. After the user has positioned the representation on the canvas, the updated canvas is stored and the user is returned to the main InfoCanvas manager web page, allowing them to add more data representations or manage existing ones.

\subsection{Monitoring}

In the background, the InfoCanvas web engine constantly monitors the information defined on the individual canvases, updating each as needed. Each canvas is published to the web as an image, allowing it to be viewed on any Internet-enabled device. The image is wrapped in a web page that is set to automatically refresh every few minutes to obtain the latest version of the user's canvas. Since the information portrayed is non-critical, a few minutes delay in the currency of the data appears acceptable.

\subsection{Customization}

The web-based version of the InfoCanvas was purposely limited to the data we thought people would most want to monitor and the representations that seemed most logical. However, we quickly realized that some users would want to define their own source of information or a representation that we had not conceived of. Therefore, we decided to allow users to create their own XML definitions of sources and representations with the aid of a tag reference we created. This allows any power-user to create extensions to the InfoCanvas framework, and enables us to quickly add new data sources and fulfill suggestions from users, ensuring the usefulness of the system does not degrade by the inability to monitor the data desired by the users.

\section{RELATED WORK}

Our approach is similar to that of the InformativeArt[9] project that uses LCD panels as ambient displays to depict email traffic flow or web site usage. Their displays, however, present only one, predetermined source of information and modify particular aspects of predesigned abstract art. Conversely, allowing end- 
users to express their own creative designs is a key part of our effort.

A slightly different approach is that of Kandinsky[1]. This system uses a repository of images, indexed by textual descriptions, primarily to build aesthetic collages that also convey information as a bonus. On the other hand, the InfoCanvas looks to support less abstract visualizations and assist the user in monitoring a large amount of information simultaneously. Our focus is on information conveyance without the absoluteness of a direct, textual representation.

Microsoft Research has developed a tool called SideShow[4] that aims to achieve the same basic goals as the InfoCanvas. In their implementation, the Windows desktop is enhanced by a panel along the side. The panel displays an overview of information that the user has specified to monitor, and allows the user to tool-tip over information to receive more details. Information is represented in a rather direct form, utilizing mostly textual and numeric output. The InfoCanvas differs from this approach in utilizing an abstract representation of the data and enabling the user to have greater control in personalizing their display. The basic design of the InfoCanvas is for use on a secondary display, whereas SideShow is primarily designed to run on the user's main display, with movement to a second display or a PDA as an option.

The Unicast system, developed by Accenture, allows for users to subscribe to "information modules" and present the information on a secondary display [6]. While running, Unicast cycles through the modules the user selected and presents the information for a few seconds. Closely related to this is the "What's Happening" desktop application, which presents community news and calendar events broadcast by a central server $[12,13]$. What's Happening provides a small, corner-of-the-display window that presents (in a cycling manner) information items of interest to the user. Again, the InfoCanvas differs from these approaches in the use of an abstract representation and the emphasis on letting the user define the information they want to monitor with greater granularity. The InfoCanvas also eliminates the cycling behavior, presenting information in a possibly calmer manner.

Another approach is to create physical devices that represent information through visual and/or aural effects. Examples of this approach are the Information Percolator[3] and the Water Lamp[11]. These types of displays are highly abstract in their method of representing data, but they are limited to representing only a few data sources at any one time, making it a lowbandwidth display. The InfoCanvas, through use of an LCD display, is able to convey a much greater amount of information through a single device.

\section{SYSTEM ARCHITECTURE}

This version of the InfoCanvas was implemented as a set of XML-driven Java Servlets and a Java application. The servlets serve as the front-end for the system, providing the web-based canvas management interface. The Java application is a continuously running background process on the same machine as the servlets that monitors information and updates the canvases. This centralized architecture was used to facilitate initial evaluation with the system, allowing users to try the InfoCanvas without having to install any additional software or updates as it evolved. The decision was made to use Java because we could rapidly build prototypes of the system and the Java 2D graphics capabilities allow us to easily create and modify images.

\subsection{Web Front End}

The servlets provide the user interface for managing a canvas. Each major function of the interface, such as specifying information, creating a representation, placing the representation, and removing information from the canvas are all separate servlets. These servlets store each canvas as an XML file. This was chosen over a database-based approach for simplicity. The XML file specifies what data the user wants to monitor and how to represent it on the canvas by defining data harvesters and data representers.

\subsection{Data Harvesters}

The information on a canvas is monitored by a set of data harvesters. Each data-driven element of the canvas has one harvester associated with it. A wide variety of data harvesters have been created, each monitoring a different data source such as stock quotes, sports scores, or even incoming emails. The parameters of each harvester define exactly what is to be harvested, such as the location for a weather forecast or traffic condition, and is stored as part of the XML canvas file.

An example of the XML definition for a weather forecast harvester is shown in Listing 1. It defines the input parameter needed from the user to retrieve the forecast for the target location, the URL for the web page containing the data, the regular expressions for extracting the forecasted high temperature and condition, and the default label to use in describing what information an instance of the harvester represents.

\subsection{Data Representers}

Each data harvester has one or more associated data representer. The data representer is a method of specifying how to create a pictorial representation of information and an optional condition determining when it should apply. There are different types of data representers, each one applying an effect to a graphical representation of the data. For example, one data representer adds an iconic image to the representation, while another rotates it based upon the data value being monitored, and yet another fills a portion based upon the data. Applying combinations of these in sequence allows for a large variety of representations to be quickly created.

For example, Listing 2 shows an excerpt from a definition for representing the forecasted weather condition. The first rule looks for the word "cloudy" in the forecast, and when present, adds a cloud image to the representation. The second rule then looks for the word "rain" in the forecast, and adds an image of rain drops when appropriate. Thus when the forecast calls for "partly cloudy with a $50 \%$ chance of rain," the InfoCanvas will display both clouds and rain images as part of the representation of the weather forecast. This demonstrates that complex representations can be created through a combination of representations. While this affords the user the ability to customize their representation, it does not completely answer the problem of end-user programming. For example, creating a representation that is different for a $10 \%$ chance of rain than for a $90 \%$ chance is hard to achieve with our existing tool. However, short of creating a specialized language for the InfoCanvas, we believe that the current ability to specify representations of information is sufficient in most cases. 
Listing 1. XML definition for the weather forecast harvester

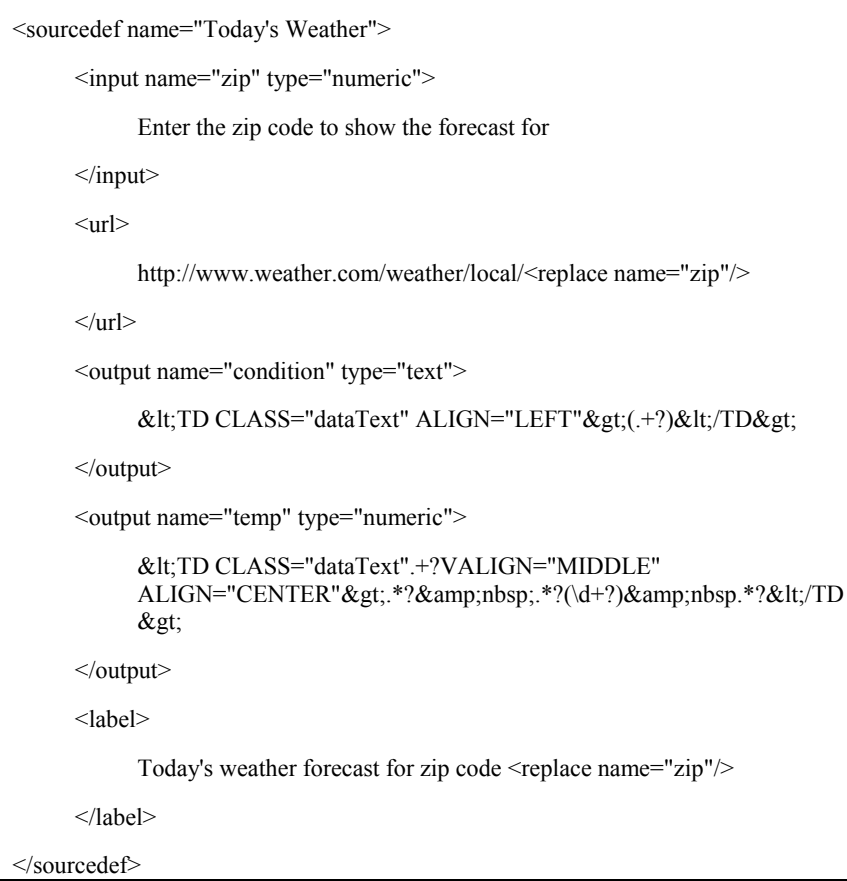

Listing 2. XML definition for representing the forecasted weather condition

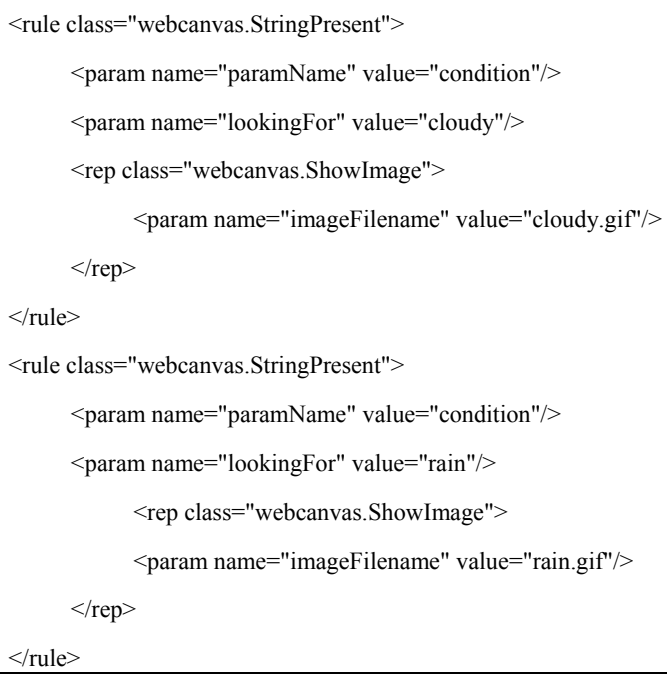

\subsection{Background Monitor}

The last piece of the InfoCanvas is the background monitor, which runs as a Java application on the web server, periodically reading in an XML canvas definition, harvesting the data specified, and then generating the actual canvas image to represent the current state of all the data.

\section{STATUS AND CONCLUSION}

We have presented the InfoCanvas, a unique tool for allowing people to stay aware of information that is meaningful to them. It allows users to monitor peripheral awareness information in a calm, ambient, abstract, personally meaningful, and hopefully eye-pleasing manner. The need for such a tool has been clearly shown by the increase in the amount of information available today and all the different competing demands for people's attention and time.

We are now deploying the system to trial users, and the next phase of the research will focus on conducting a study of the InfoCanvas in use. The goal is to determine the ability of the InfoCanvas to effectively assist people in monitoring peripheral awareness information. This will allow us to make an assessment of what information users want to monitor and how they want it to be represented.

We also plan to explore the notion of predefined canvases. These canvases will be created by artists and hopefully will be an attractive option to less artistically talented users. One challenge is to still allow some level of customization with such predefined canvases.

Future work on the InfoCanvas will involve creating a more fullfeatured version of the system. This future version should enable a wider variety of information to be monitored (such as to-do lists, people's on-line presence, schedules, printer queues, etc.), and should support the creation of much more flexible and richer representations. One problem as we move closer towards the ability to "paint information" is avoiding a large learning threshold, such as a complex interface (like that of commercial tools such as Adobe PhotoShop) or a special end-user programming language (such as AccessBasic). Since peripheral information is not of critical importance, we do not know whether we can expect users to exert the same energy in learning how to fully use a tool as they would for a critical task. Measuring how willing users are to learn about the InfoCanvas and its XMLdriven language would allow us to determine how an end-user programming approach would be received. It is possible that monitoring peripheral information would be important enough to warrant the learning of a specialized language by the user.

Lastly, we plan to explore a mixture of direct and abstract representations. For some information, being able to see textual or numeric output is more desirable than a pictorial representation (as in a baseball score). However, integrating highly direct representations in an aesthetically-pleasing manner to a canvas will be a challenging task.

Overall we have found the InfoCanvas to be a useful tool personally and look we are continuing to explore the space and move towards the vision of a fluid, artistic tool that can seamlessly integrate art and information in a highly personalized manner.

\section{ACKNOWLEDGMENTS}

This research has been supported in part by a grant from the National Science Foundation, IIS-0118685. Thanks to the Information Interfaces group at Georgia Tech for all the support, and Autumn for superb editing. 


\section{REFERENCES}

[1] Fogarty, J., Forlizii, J., and Hudson, S.E., "Aesthetic Information Collages: Generating Decorative Displays that Contain Information", Proceedings of UIST 2001Conference, (Orlando, Florida, November 2001), pp. 141-150.

[2] Grudin, J., "Partitioning Digital Worlds: Focus and Peripheral Awareness in Multiple Monitor Use", Proceedings of the ACM CHI 2001 Conference, (Seattle, WA, April 2001), pp. 458-465.

[3] Heiner, J.M., Hudson, S.E., and Tanaka, K., "The Information Percolator: Ambient Information Display in a Decorative Object", Proceedings of UIST 1999 Conference, (Asheville, NC, 1999), pp. 141-148.

[4] J.J. Cadiz, Venolia, G.D., Jancke, G., and Gupta, A., "Sideshow: Providing Peripheral Awareness of Important Information", Technical Report MSR-TR-2001-83, Microsoft Research, September 2001.

[5] Lyman, P. and Varian, H.R., "How Much Information? (Executive summary)", www.sims.berkeley.edu/how-much-info/, University of California, Berkeley, School of Information Management and Systems.

[6] McCarthy, J.F., Costa, T.J., and Liongosari, E.S., "UniCast, OutCast \& GroupCast: Three Steps Toward Ubiquitous, Peripheral Displays", Proceedings of Ubicomp 2001 Conference, (Atlanta, Georgia, September 2001), pp. 332-345.

[7] Miller, T. and Stasko, J., "The InfoCanvas: Information Conveyance through Personalized, Expressive Art", In the ACM
CHI 2001 Extended Abstracts, (Seattle, WA, April 2001), pp. 305-306.

[8] MyYahoo! web site. http://my.yahoo.com/

[9] Redstrom, J., Skog, T., and Hallnas, L., "Informative Art: Using Amplified Artworks as Information Displays", Proceedings of DARE 2000, (Elsinore, Denmark, April 2000), pp. 103-114.

[10] Weiser, M., and Brown, J., "Designing Calm Technology", PowerGrid Journal, v1.01, (July 1996), (see: http://powergrid.electiciti.com/1.01).

[11] Wisneski, C., et al., “Ambient Displays: Turning Architectural Space into an Interface between People and Digital Information", Proceedings of the International Workshop on Cooperative Buildings, (Darmstadt, Germany, Feburary 1998), pp. 22-32.

[12] Zhao, A.Q. and Stasko, J.T., "What's Happening? The Community Awareness Application", In the ACM CHI 2000 Extended Abstracts, (The Hague, Netherlands, April 2000), pp. 253-254.

[13] Zhao, A.Q. and Stasko, J.T., "What's Happening?: Promoting Community Awareness Through Opportunistic, Peripheral Interfaces", Proceedings of AVI 2002, (Trento, Italy, May 2002). 